\title{
Novel Structures and Properties of Gold Nanowires
}

\author{
Baolin Wang, ${ }^{1}$ Shuangye Yin, ${ }^{1}$ Guanghou Wang, ${ }^{1}$ Alper Buldum, ${ }^{2}$ and Jijun Zhao ${ }^{1,2,3}$ \\ ${ }^{1}$ National Laboratory of Solid State Microstructures, Nanjing University, \\ Nanjing 210093, China \\ ${ }^{2}$ Department of Physics and Astronomy, University of North Carolina at Chapel Hill, \\ Chapel Hill, North Carolina 27599 \\ ${ }^{3}$ International Centre for Theoretical Physics, P.O. Box 586, Trieste 34100, Italy
}

(Received 24 October 2000)

\begin{abstract}
The structures of free-standing gold nanowires are studied by using molecular-dynamics-based genetic algorithm simulations. Helical and multiwalled cylindrical structures are found for the thinner nanowires, while bulk-like fcc structures eventually form in the thicker nanowires up to $3 \mathrm{~nm}$ in diameter. This noncrystalline-crystalline transition starts from the core region of nanowires. The vibrational, electronic, and transport properties of nanowires are investigated based on the optimal structures. Bulklike behaviors are found for the vibrational and electronic properties of the nanowires with fcc crystalline structure. The conductance of nanowires generally increases with wire diameter and depends on the wire structure.
\end{abstract}

DOI: 10.1103/PhysRevLett.86.2046

Metallic nanowires have been intensively studied because of the fundamental interests in low-dimensional physics and technological applications as molecular electronic devices [1-21]. Most of the previous studies on metallic nanowires are focused on the nanoscale atomic necks formed by point contact between two metallic nodes [1-17]. In recent years, long metallic nanowires with well-defined structures and a diameter of several nanometers have been fabricated by using different methods [18-21]. For example, stable gold nanobridge with $0.8-3 \mathrm{~nm}$ in thickness and $5-10 \mathrm{~nm}$ in length were produced by electron-beam irradiation of gold (001) oriented thin film [18]. Cu nanorods were deposited on substrate and characterized [19]. Recently, suspended gold nanowires with $6 \mathrm{~nm}$ in length and diameters down to $0.6 \mathrm{~nm}$ were made and the novel helical multishell structures are observed [21]. Therefore, detailed studies on the structures and properties of such long and nearly free standing ultrathin nanowires are needed. Previous theoretical works include melting behavior of $\mathrm{Pb}$ nanowires [22], noncrystalline structures of $\mathrm{Al}$ and $\mathrm{Pb}$ nanowires [23], structural and vibration properties of finite $\mathrm{Au}$ nanowires [24], and electronic structures and conductance of Al nanowires [25]. However, the size dependence of atomic structure, vibrational spectra, electronic states, and conductance, as well as the structural effect on electronic and transport properties of metallic nanowires are still unclear.

In this Letter, we have systematically studied the structures and properties of gold nanowires with diameters from 0.5 to $3.0 \mathrm{~nm}$. The nanowires with sufficient length are modeled by a supercell with a one-dimensional (1D) periodical boundary condition along the wire axis direction. The length of the supercell is chosen as $1.221 \mathrm{~nm}$, which is a reasonable compromise between discovering the helicity in the 1D direction and avoiding the break of nanowires into clusters upon relaxation. The interaction between
PACS numbers: 61.46.+w, 68.65.-k, 72.80.Rj, 73.61.- $\mathrm{r}$

gold atoms is modeled by a glue potential [26], which has successfully described the gold surfaces [26] and nanoclusters [27]. For structural optimization, we adopt a genetic algorithm [28] based on molecular dynamics (MD) relaxation. At the beginning, a number of initial configurations are generated at random with arbitrary orientation. Any two candidates in the population can be chosen as parents to generate a child configuration through a proper mating process [28]. The generated child is then relaxed by MD quenching and selected to replace its parent in the population if it has different geometry and lower energy.

Based on the optimized structures, density functional theory (DFT) electronic structure calculations are performed by using the DMOL program [29] with effective core potential. The density functional is treated by local density approximation with a Wang-Perdew exchangecorrelation functional [30]. A minimal numerical basis is used and the Brillouin zone is sampled by $\Gamma$ point. We adopt the Harris functional approximation [31] in the DFT calculation, which has been used to study large Ag nanoclusters [32]. We also investigate the transport properties by using Landauer formalism with the surface Green's function matching method [33,34]. The electronic structures of gold nanowires are described by an orthogonal nearest-neighbor $s$-orbital tight-binding Hamiltonian, which has been successfully applied to gold point contacts [15].

Several typical structures of gold nanowires are shown in Fig. 1. Helical $(D \leq 0.6 \mathrm{~nm})$, multiwalled cylindrical $(D=1.0 \sim 2.2 \mathrm{~nm})$, and fcc-like $(D>2.2 \mathrm{~nm})$ structures are found for nanowires with different sizes. In the nanowires below $0.6 \mathrm{~nm}$, the most stable structures are helical packing composed of several identical spiral strands (A1, A2 in Fig. 1). Similar helical structures have been predicted for $\mathrm{Pb}$ and $\mathrm{Al}$ nanowires [23] and recently observed in the gold nanowires [21]. As $D>0.6 \mathrm{~nm}$, the nanowire structures switch to the multiwalled 


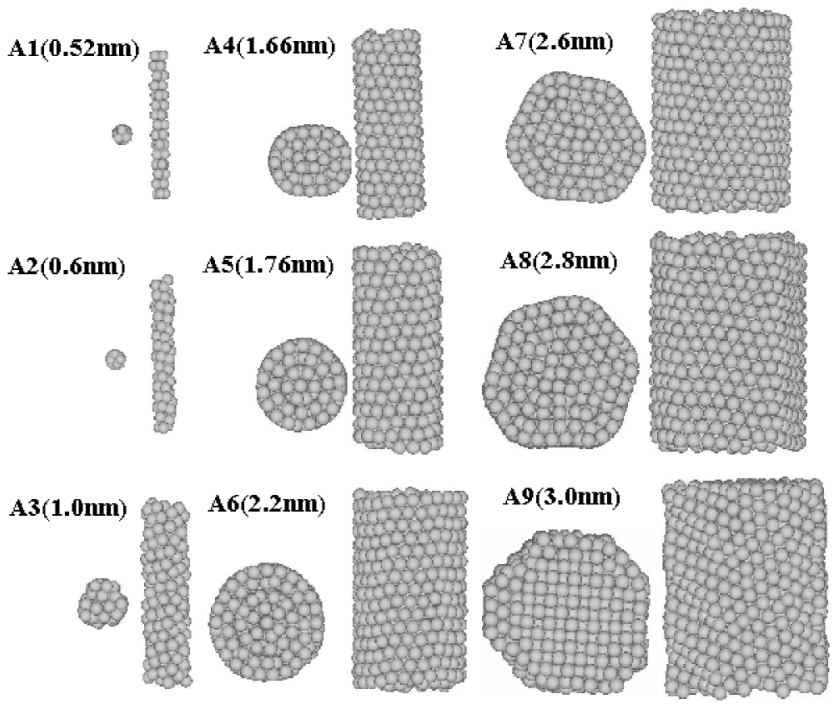

FIG. 1. Morphology of some selected gold nanowires with a diameter from 0.5 to $3.0 \mathrm{~nm}$. The diameter and correspondent symbols (A1-A9) for each nanowire are presented.

cylindrical form (A3-A6). These multiwalled structures are composed of several close-packed curved layers, which can also be viewed as coaxial tubes formed by the helical atomic rows coiling around the wire axis. The obtained surface packing structure, so called "curved surface epitaxy," seems to be a common feature in metal nanowires $[23,24]$. To reveal more possible chiralities in nanowires, variable supercell length is required. However, the helical multishell structures obtained from our present simulations agree well with experiment [21]. It is also worthy to note that the nanowire with $D=1.0 \mathrm{~nm}$ has a double-walled structure and that with $D=1.66 \mathrm{~nm}$ has a triple-walled structure, while experimental study has observed double-walled for $D=0.6-1.0 \mathrm{~nm}$ and triple-walled for 1.1-1.3 $\mathrm{nm}$ [21].

As the nanowire size further increases, the crystalline fcc structures gradually form in the core region of the nanowire with more than four layers in the radial direction (A7, A8). To illustrate such a transition, we present the atomic cross-section projections of $\mathrm{A} 7, \mathrm{~A} 8$, and $\mathrm{A} 9$ nanowires in Fig. 2. One can find that the A7 and A8 wires have the crystalline cores surrounded by the outer four continuous circles, i.e., noncrystalline curved surfaces. These structures can be considered as being very thin crystalline wires coated with few layers of reconstructed noncrystalline surface. Accordingly, in the recent experiment, the gold nanowires larger than $1.5 \mathrm{~nm}$ are composed of fcc core covered by reconstructed (111)-like close-packed structure [21]. In contrast to A7 and A8, the projection array of A9 wire in Fig. 2 is very regular except for a few dislocated dots in the outmost surface. In other words, the gold nanowires up to $3 \mathrm{~nm}$ are rather crystalline and the noncrystalline structure can exist only in the outmost surface layer.

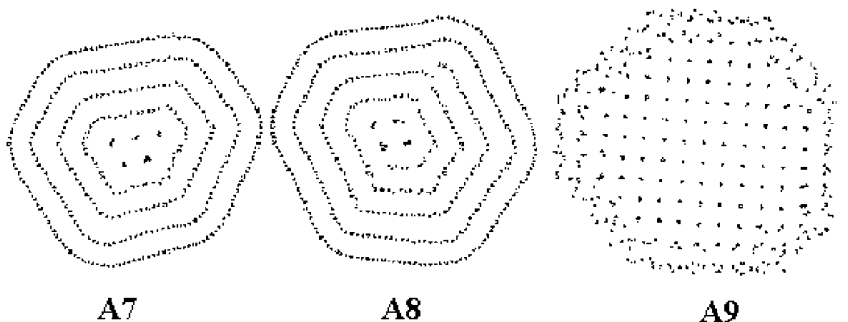

FIG. 2. Atomic cross-section projections for A7, A8, and A9 wires.

The structural properties of nanowires can also be characterized by the angular correlation functions (ACFs) shown in Fig. 3. For those thinner wires, the ACFs demonstrate multipeaks with rather wide distributions since the noncrystalline structures don't have any definite bond angle. As nanowire size increases, the ACF spectrum gradually comes into being three peaks centered at $60^{\circ}$, $90^{\circ}$, and $120^{\circ}$, corresponding to the bond angle in the bulk fcc structures. At the thickest wire A9, these three peaks become sharp and dominant in the ACF. From the above results, we propose that the free standing nanowires with diameter larger than $3.0 \mathrm{~nm}$ should be nearly crystalline.

Figure 4 shows vibrational densities of states for A1-A9 nanowires obtained by diagonalizing the dynamical matrix

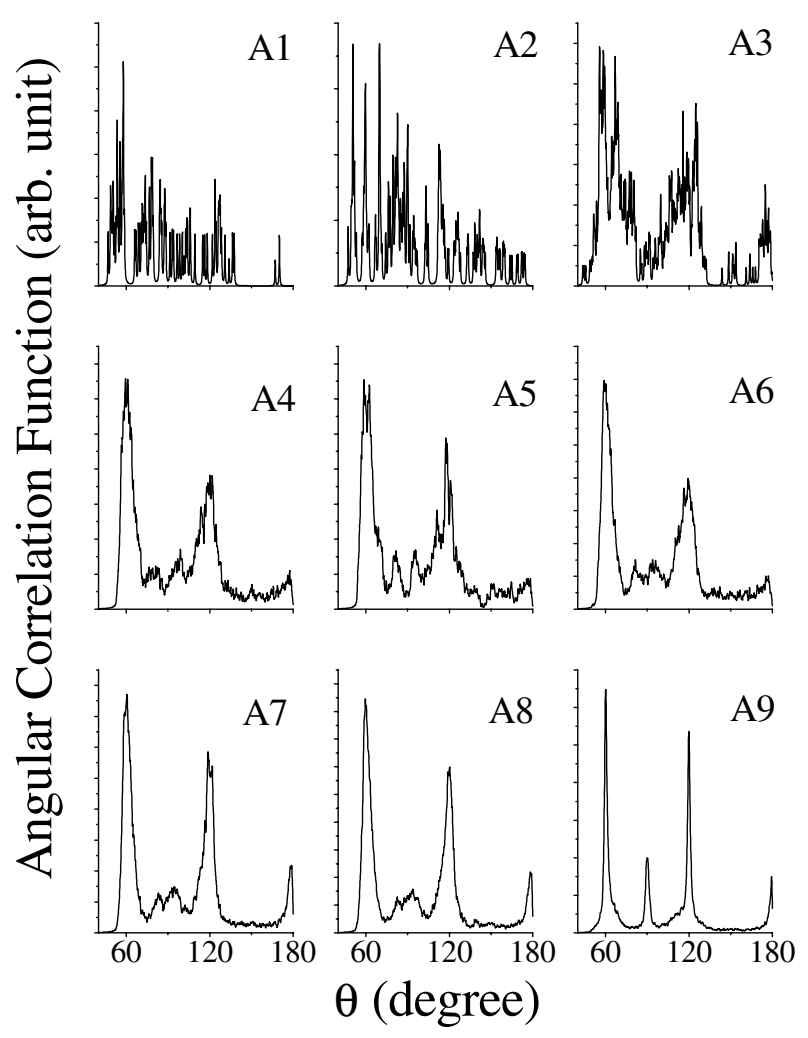

FIG. 3. Angular correlation functions (ACF) of several gold nanowires shown in Fig. 1. A9 is already very close to the ACF of a perfect fcc solid. 
for the equilibrium structures with glue potential. The vibrational spectrum of A9 wire has a maximum frequency of about $4.7 \mathrm{THz}$ and two other peaks located at 2.3 and $3.7 \mathrm{THz}$. This behavior is similar to that of bulk fec gold [35]. An additional peak around $4.2 \mathrm{THz}$ is observed in A4-A8 nanowires. This peak gradually decreases as the nanowire radius increases and finally disappears in the A9 nanowires with fcc structure. Therefore, this peak is obviously related to the outer curved surface in $\mathrm{A} 4-\mathrm{A} 8$ and may be attributed to vibration modes between the atomic layers of curved surface epitaxy. We also find that the position and the shape of the first peak around $2.3 \mathrm{THz}$ do not sensitively change for all those wires except for A1 and A2. In contrast to the bulklike spectra in A3-A9, the thinnest wires with helical structure (A1 and A2) have many discrete vibrational bands due to the absence of spatial symmetry. The maximal vibration frequency is found to be $13.0 \mathrm{THz}$ for $\mathrm{A} 1$ and $8.5 \mathrm{THz}$ for A2. They are much higher than those for the multiwalled and fcc structures, but comparable to the frequency calculated for monatomic gold chains and dimer [36].

In Fig. 5, we present the size evolution of the electronic density of states of nanowires towards the bulk limit. The very thin nanowire (A2) shows a moleculelike feature and its DOS has many sharp and discrete peaks. In A3 $(D=1.0 \mathrm{~nm})$, the discrete levels gradually overlap with each other and start to form continuous electronic bands. The shape of the DOS of A3-A9 nanowires (1.0-3.0 nm)

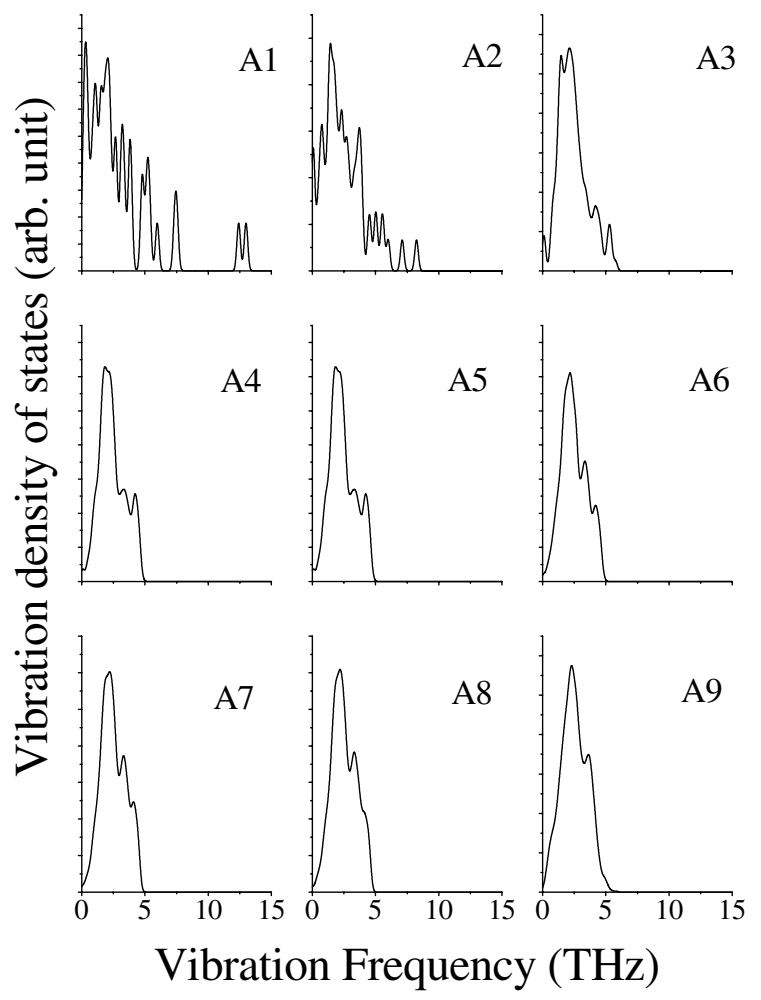

FIG. 4. Vibrational spectra of gold nanowires for A1-A9 wires. does not sensitively depend on wire diameter, while the width of the electronic band gradually narrows as the wire become thicker. The band narrowing can be understood by the two-dimensional (2D) quantum confinement effect in nanowires, i.e., the amplitude of energy level broadening should be inversely proportional to the dimension of the 2D quantum well. Up to the A9 wire, the width of the electronic band is already very close to the bulk value and the DOS considerably resembles the bulk one. However, the bulk DOS shows some fine structures and the DOS spectrum for A9 nanowires looks like the average of the bulk DOS. For the A9 wire, there is still a large portion of atoms sitting on the surface, which may modify the well-defined peaks of bulk $d$ bands. Thus, we argue that the bulk electronic properties have been roughly achieved in gold wire thicker than $3 \mathrm{~nm}$, although some detail differences still exist. In a previous DFT calculation of gold nanoclusters [37], the cluster DOS evolves rather quickly from a discrete behavior for the small clusters like $\mathrm{Au}_{13}$ to a "bandlike" appearance in the $\operatorname{Au}_{147}(D=1.7 \mathrm{~nm})$. In addition, the size evolution behavior of electronic properties in Fig. 5 is comparable to that found in ACF (Fig. 3) and vibrational spectra (Fig. 4). In particular, the vibrational and electronic properties of the nanowires with crystalline structure (A9) are already very close to bulk behavior. We expect future experiments to validate our arguments.

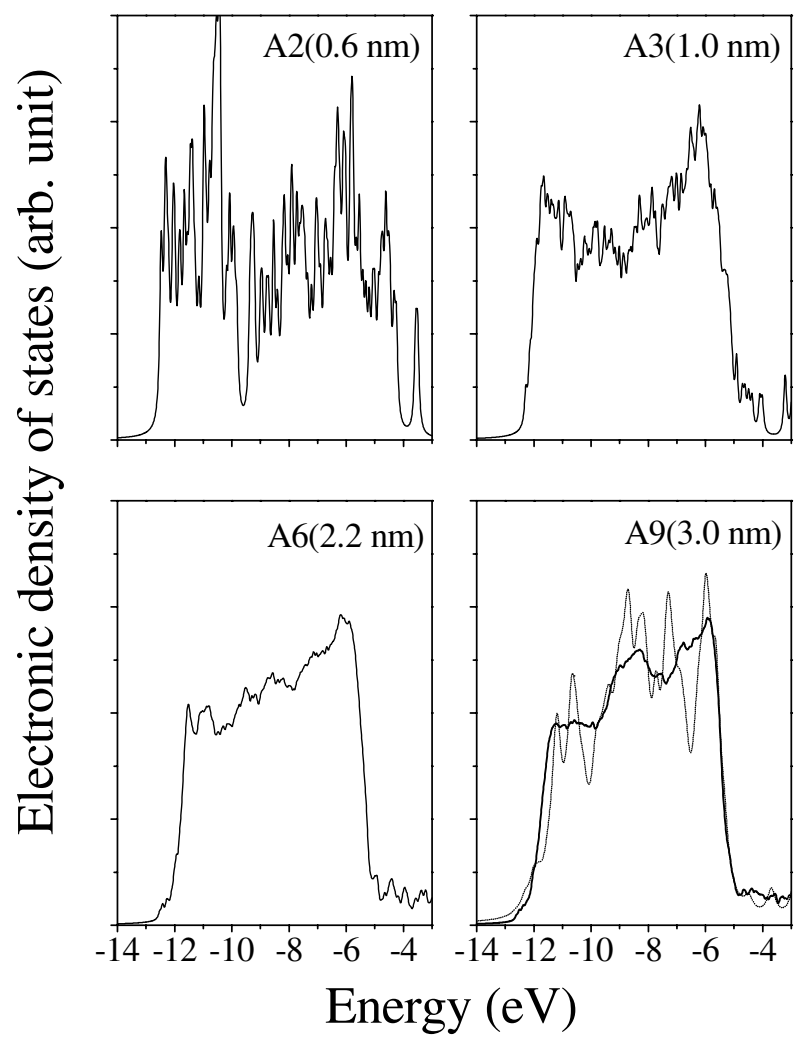

FIG. 5. Electronic density of states (DOS) of gold nanowires A2, A3, A6, A9 compared with DOS of bulk gold (dashed line). 


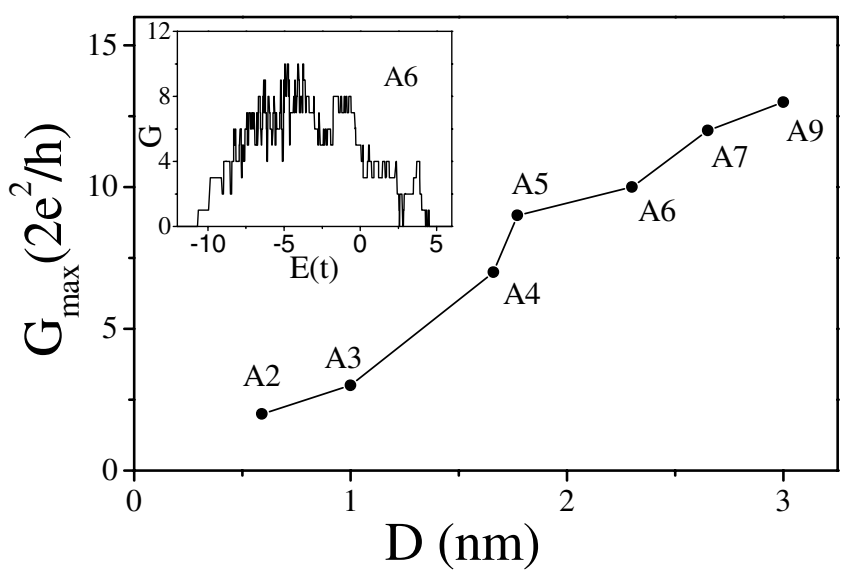

FIG. 6. The maximum value of conductance as a function of diameter of gold nanowires. The inset figure shows a typical variation of conductance $(G)$ of gold nanowire (A6) with the energy $E$ scaled by the tight-binding nearest-neighbor hopping integral $t$. The maximum conductance of each wire (A2-A9) has an integer value and is represented by a filled circle.

The maximum values of conductance (or maximum number of conducting channels) of the gold nanowires (A2-A9) and a typical conductance of nanowires (A6) are presented in Fig. 6. In general, the number of conducting channels increases linearly with nanowire diameter. This can be attributed to the increase of available conducting states as the number of atoms in the nanowire increases. Previous condunctance calculations on jellium wires and quantum constriction showed a stepwise increase of conductance with a continuous increase of diameter $[13,25,38]$. Our conductance calculations are performed on a finite number of nanowires and do not show this stepwise increase. The atomic structure and symmetry of nanowires also play a role in determining conductance. For example, the highly symmetric A5 wire with fewer defects has a particular enhanced conductance. Moreover, in Fig. 6, the linear increase of $G_{\max }$ with $D$ from A3 to A7 (noncrystalline multiwalled structures) drops at the A9 wire in which the fcc structure prevails. Such structural dependence of conductance can be verified by experiments and further theoretical investigations. Preliminary constant-force MD simulations on these nanowires also show that the A5 wire is particularly strong upon external pulling. Our further works including the effect of $d$ electrons and the mechanical and transport properties of such "magic-sized" nanowire are still underway and the results will be reported elsewhere.

From the above discussions, the following conclusions can be made. (1) Helical, multiwalled, cylindrical, and fcc structures are found for the gold nanowires. (2) The transition from noncrystalline to fcc starts from the core region and the nanowires with $D \geq 3.0 \mathrm{~nm}$ are almost crystallines. (3) Vibrational properties of nanowires show significant size dependence: the smallest nanowires are moleculelike, while the spectra for large wires are close to bulk behavior. (4) The band width of electronic DOS gradually decreases as the wire becomes thicker and the electronic structures of nanowires with fcc structure show considerable bulk like feature. (5) The conductance of nanowires generally increases with wire size and the geometric structure has a certain influence on the transport properties.

This work is financially supported by NNSF of China (No. 29890210) and U.S.ARO (No. DAAG55-98-1-0298). We thank Professor E. Tosatti, Professor J.P. Lu, and Professor C.Z. Wang for discussions and computational support from the North Carolina Supercomputer Center.

[1] U. Landman et al., Science 248, 454 (1990).

[2] C. L. Muller et al., Phys. Rev. Lett. 69, 140 (1992).

[3] N. Agrait et al., Phys. Rev. B 47, 12345 (1993).

[4] J. I. Pascual et al., Phys. Rev. Lett. 71, 1852 (1993).

[5] L. Olesen et al., Phys. Rev. Lett. 72, 2251 (1994).

[6] J. I. Pascual et al., Science 267, 1793 (1995).

[7] J. M. Krans et al., Nature (London) 375, 767 (1995).

[8] G. Rabio et al., Phys. Rev. Lett. 76, 2302 (1996).

[9] A. Stalder, U. Durig, Appl. Phys. Lett. 68, 637 (1996).

[10] U. Landman et al., Phys. Rev. Lett. 77, 1362 (1996).

[11] C. Untiedt et al., Phys. Rev. B 56, 2154 (1997).

[12] J. L. Costa-Kramer et al., Phys. Rev. Lett. 78, 4990 (1997).

[13] E. Tekman and S. Ciraci, Phys. Rev. B 39, 8772 (1989).

[14] A. I. Yanson et al., Nature (London) 395, 783 (1998).

[15] A. M. Bratkovsky et al., Phys. Rev. B 52, 5036 (1995); T. N. Todorov and A.P. Sutton, Phys. Rev. B 54, 14234 (1996).

[16] A. Nakamura et al., Phys. Rev. Lett. 82, 1538 (1999).

[17] H. Hakkinen et al., J. Phys. Chem. B 103, 8814 (1999).

[18] Y. Kondo and K. Takayanagi, Phys. Rev. Lett. 79, 3455 (1997).

[19] I. Lisiecki et al., Phys. Rev. B 61, 4968 (2000).

[20] W. S. Yun et al., J. Vac. Sci. Technol. A 18, 1329 (2000).

[21] Y. Kondo and K. Takayanagi, Science 289, 606 (2000).

[22] O. Gulseren et al., Phys. Rev. B 51, 7377 (1995).

[23] O. Gulseren et al., Phys. Rev. Lett. 80, 3775 (1998).

[24] G. Bilalbegovic, Phys. Rev. B 58, 15412 (1998).

[25] F. Di Tolla, Surf. Sci. 454, 947 (2000).

[26] F. Ercolessi et al., Phys. Rev. Lett. 57, 719 (1986).

[27] F. Ercolessi et al., Phys. Rev. Lett. 66, 911 (1991).

[28] Y. H. Luo et al., Phys. Rev. B 59, 14903 (1999).

[29] DMOL/DSOLID is a DFT package based localized basis distributed by MSI. B. Delley, J. Chem. Phys. 92, 508 (1990).

[30] Y. Wang and J. P. Perdew, Phys. Rev. B 43, 8911 (1991).

[31] J. Harris, Phys. Rev. B 31, 1770 (1985).

[32] D. R. Jennison et al., J. Chem. Phys. 106, 1856 (1997).

[33] S. Datta, Electronic Transport in Mesoscopic Systems (Cambridge University Press, Cambridge, 1995).

[34] F. Garcia-Moliner and V. R. Velasco, Phys. Rep. 200, 83 (1991); M. B. Nardelli, Phys. Rev. B 60, 7828 (1999).

[35] J. W. Lynn et al., Phys. Rev. B 8, 3493 (1973).

[36] D. Sanchez-Portal et al., Phys. Rev. Lett. 83, 3884 (1999).

[37] O. D. Haberien et al., J. Chem. Phys. 106, 5189 (1997).

[38] A. Garcia-Martin et al., Phys. Rev. B 54, 13448 (1996). 